\title{
Supervisão: estilos, satisfação e sintomas depressivos em estagiários de psicologia
}

\author{
Ana Catarina Marques Ribeiro de Figueiredo - Psiquilíbrios \\ Susana Margarida Gonçalves Caires Fernandes - Universidade do Minho ${ }^{1}$ \\ Carla Cristina Esteves Martins - Universidade do Minho \\ Vera Lúcia Moreira Ramalho - Psiquilíbrios
}

\begin{abstract}
Resumo
A figura do supervisor assume-se como um dos principais factores protectores da experiência de estágio, emergindo como uma importante fonte de suporte emocional e/ou como um "amortecedor" do impacto gerado pela transição universidade-mundo do trabalho. Visando estudar a relação entre os estilos de supervisão, os níveis de satisfação do estagiário (em relação aos supervisores da universidade e da instituição de estágio) e a sua eventual relação com sintomatologia depressiva, foi realizado um estudo com 83 estagiários de Psicologia da Universidade do Minho, avaliados no final do seu estágio, através do Inventário de Estilos Supervisivos, do Questionário da Qualidade da Supervisão e do Inventário da Depressão de Beck. Os resultados revelam uma concordância na forma como estes avaliam ambos os supervisores em termos de estilo supervisivo e satisfação com a supervisão, surgindo uma maior satisfação associada a um estilo atractivo e orientado para a tarefa. No caso do supervisor da universidade, a periodicidade regular do seu acompanhamento emerge como uma variável importante, associando-se a maiores níveis de satisfação. Não foram encontradas correlações entre qualquer uma dessas variáveis e sintomatologia depressiva.

Palavras-chave: Estilos supervisivos; Satisfação com a supervisão; Sintomatologia depressiva.
\end{abstract}

\section{Supervision: supervisory style, supervisee satisfaction and depressive symptoms in psychology students}

\begin{abstract}
In this paper the supervisor is assumed as having a determinant role on the quality of the trainee's experience, acting as an important source of emotional support and as a "buffer" of the impact caused by the university-to-work transition. In an attempt to explore possible relationships between the supervisory styles, the supervisee's satisfaction (regarding the university and the field supervisor) and depressive symptomatology the authors carried out a study, at the University of Minho, involving 83 psychology trainees evaluated at the end of their practicum. The study was based on the Supervisory Styles Inventory, the Supervisee Satisfaction Questionnaire and the Beck's Depression Inventory. Results show conformity in the way trainees perceive both supervisors in terms of supervisory style and of satisfaction with their guidance and support. Greater satisfaction levels are related to the combination of an attractive and task oriented style. Concerning the university's supervisor, the periodicity of the meetings with this teacher emerges as an important variable: higher levels of satisfaction correspond to a more regular supervision. No connection was found in terms of these two variables and depressive symptoms.
\end{abstract}

Keywords: Supervisory style; Supervisee satisfaction; Depressive symptoms.

Supervisão: estilos, satisfação e sintomas depressivos em estagiários de psicologia

O termo transição significa acto ou efeito de passar de um estado, período, assunto ou lugar para outro (Dicionário de Língua Portuguesa, 2003) - numa palavra, mudança. Schlossberg define transição como "[...] the changes - good or bad, expected or unexpected - that unsettle our lives, shake us up, and take some adjusting over time" (1989, p. $\mathrm{xv}$ ). Segundo esse autor, uma transição como aquela que tem lugar na passagem da universidade para o mundo profissional implica mudanças no nível das rotinas, papéis, relacionamentos interpessoais e da percepção acerca de si e do mundo.

Como nas demais transições, o estágio é vivenciado com alguma apreensão e expectativa pelos seus principais intervenientes - os estagiários. Para Caires, tratase de um "[...] período de intensa exploração e descoberta de si próprio, dos outros e dos contextos onde passará a movimentar-se" (2003, p. 137), pautado por uma

${ }^{1}$ Endereço para correspondência:

Universidade do Minho - Instituto de Educação e Psicologia - Departamento de Psicologia

Câmpus de Gualtar, 4.710 - Braga - Portugal

E-mail: caires@iep.uminho.pt 
multiplicidade de cognições e afectos que, pela sua diversidade, intensidade e complexidade, fazem do estágio um dos momentos mais ricos e desafiantes do percurso dos alunos do ensino superior. Alguns desses desafios prendem-se com a adopção de novos papéis, a adaptação a um novo contexto com rotinas, regras e "padrões" distintos do meio académico, bem como o afastamento da rede social construída ao longo dos primeiros anos do curso. A promoção de competências de empregabilidade e desenvolvimento de destrezas na sua área profissional, o desenvolvimento de competências sociais e interpessoais, ganhos no nível da sua auto-estima e sentido de autoeficácia, ou os maiores níveis de auto-conhecimento atingidos surgem, geralmente, em resultado do confronto com tais desafios (Caires, 2001; Malglaive, 1997; Ryan, Toohey \& Hughes, 1996).

Apesar dos múltiplos ganhos decorrentes dessa experiência, são também várias as dificuldades vivenciadas, surgindo o estágio como responsável por alguns desequilíbrios nas esferas física e psicossocial. Entre estes, alguma literatura destaca os elevados níveis de estresse e ansiedade experienciados, o acentuado desgaste físico e emocional, as alterações nos padrões de sono e de apetite, ou alguns sintomas depressivos (Caires, 2001; Capel, 1997; Matos \& Costa, 1993).

\section{A relação de supervisão}

No âmbito das dificuldades vivenciadas pelos estagiários nesse período de transição, a relação mantida com o(s) supervisor(es) emerge como uma dimensão central, sendo apontada por alguns autores como um factor determinante da qualidade dessa experiência (Alarcão \& Tavares, 2003; Caires, 2001, 2003; Hawkey, 1997; Ryan e cols., 1996). Segundo Soares, é

[...] no seio desta matriz relacional que se encontram as condições propícias à exploração, à expressão e à integração das experiências e o suporte para os riscos envolvidos no ensaio de novas formas de pensar, sentir e agir. (1995, p. 145)

Outros autores salientam o papel do supervisor enquanto facilitador das transições ecológicas que têm lugar durante o estágio, reforçando, também, a importância do apoio emocional a dar ao formando. Uma relação significativa, emocionalmente carregada e pautada pelo respeito mútuo, confiança e apoio é apontada como elemento-chave ao desenvolvimento pessoal e profissional dos estagiários (Alarcão \& Tavares, 2003; Caires \& Almeida, 2001; Soares, 1995).

Embora, na grande parte das situações, as relações supervisivas sejam bem sucedidas, a verdade é que, noutras, estas poderão surgir como um desafio acrescido para o estagiário. Sentimentos de impotência, insegurança, abandono ou mesmo humilhação surgem, por vezes, associados aos maiores níveis de estresse e vulnerabilidade apresentados em resultado das dificuldades vividas no seio dessa relação (Caires, 2001; Ellis, 2001; Nelson \& Friedlander, 2001). Uma das questões que daqui poderá decorrer prende-se com alguns dos factores que estarão por detrás dessa vulnerabilidade acrescida: se, por exemplo, a ausência/negligência do supervisor; se a existência de conflitos explícitos entre as duas partes (no nível pessoal, científico, ético...); o desajustamento/ insuficiência das respostas do supervisor às solicitações do estagiário; ou a desadequação das mesmas ao tipo de necessidades do estagiário (científicas, metodológicas, emocionais...). Por outras palavras: será que a forma como o supervisor aborda e responde às necessidades do estagiário e/ou o modo como dirige o processo supervisivo influencia os níveis de vulnerabilidade relatados pelos estagiários? Existirá algum estilo de supervisão que melhor se ajuste/satisfaça as diferentes necessidades do estagiário?

Friedlander e Ward, centradas na averiguação de algumas dessas questões, definem estilo de supervisão como "[...] the supervisor's distinct manner of approaching and responding to trainees and of implementing supervision" (1984, p. 541) e assumem-no como multidimensional. Segundo as autoras, é a existência de várias dimensões interrelacionadas que explica a variabilidade observada entre os supervisores, tais como: as orientações teóricas, as técnicas utilizadas pelo supervisor e o seu estilo relacional. A partir da análise de amostras distintas, três factores interrelacionados foram consistentemente apontados como fortemente associados à satisfação dos orientandos: o estilo "atractivo" do supervisor, que Friedlander e Ward entendem como o estilo de supervisão que promove um ambiente coloquial entre supervisor e estagiário (e.g. "amigável", "aberto", "flexível"); a "sensibilidade a nível interpessoal" do supervisor, que se traduz num estilo orientado para a relação e para a satisfação das necessidades do estagiário (e.g. "perspicaz", "intuitivo", "terapêutico") e, finalmente, o estilo "orientado para a tarefa", que reflecte a centração do supervisor nos conteúdos e tarefas, e nas aprendizagens dos estagiários (e.g. "objectivo", "estruturado", "pragmático").

Numa tentativa de explorar o modo como os estagiários avaliam a supervisão do seu estágio bem como o seu impacto no bem-estar desses formandos, foi desenvolvido um estudo com estagiários de psicologia da Universidade do Minho (UM), onde as suas percepções acerca dos supervisores da universidade e da instituição do estágio (IE) foram averiguadas. A satisfação relativamente à supervisão recebida, as diferenças (ou não) entre os níveis de satisfação relativos a cada um dos supervisores, a 
preferência dos estagiários por determinados estilos de supervisão ou eventuais relações entre os menores níveis de satisfação dos estagiários e a presença de sintomatologia depressiva surgiram como questões centrais. Foi igualmente averiguada a influência das variáveis género, periodicidade da supervisão, área de pré-especialização do estagiário e área de formação do supervisor da IE (psicólogo vs não-psicólogo) nas variáveis anteriores.

\section{Metodologia}

\section{Estágios da Licenciatura em Psicologia da Universidade do Minho}

$\mathrm{Na} \mathrm{UM}$, o estágio curricular ocorre no $5^{\circ}$ e último ano da licenciatura em psicologia e tem uma duração aproximada de 10 meses (um ano lectivo). O tempo passado na instituição de estágio (e.g. escola, hospital, centro de saúde, empresa, estabelecimento prisional) é complementado com as idas à universidade, uma vez por semana, onde decorrem os seminários de investigação e de intervenção e as reuniões de supervisão. Os seminários são espaços de formação, de grande grupo, reservados à apresentação e discussão de alguns suplementos teóricos e ao desenvolvimento de um projecto de investigação e outro de intervenção. Complementarmente, ocorrem as reuniões, individuais ou de pequeno grupo, com o supervisor da UM onde tem lugar a discussão e reflexão sobre as experiências desenvolvidas no local de estágio.

No que se refere à supervisão na IE, o acompanhamento é, geralmente, regular e contínuo, ocorrendo num registo diário. Nalguns casos, em razão da inexistência de profissionais de psicologia na instituição, o responsável pela supervisão dos estagiários é um técnico de outra área disciplinar (e.g. médico, assistente social, enfermeiro).

\section{Participantes}

Participaram neste estudo 83 estagiários do $5^{\circ}$ ano de psicologia da UM, de entre um universo de 121, provenientes de diferentes áreas de pré-especialização: psicologia clínica e da saúde $(\mathrm{N}=40,48,2 \%)$; psicologia da justiça e da reinserção $(\mathrm{N}=18,21,7 \%)$; psicologia escolar e da educação $(\mathrm{N}=13,15,7 \%)$; e psicologia social, comunitária e das organizações ( $\mathrm{N}=12,14,5 \%)$. O grupo é predominantemente do sexo feminino ( $\mathrm{n}=69,83,1 \%)$. Aproximadamente $3 / 4$ dos estagiários teve supervisão regular na universidade (semanal, $\mathrm{n}=45 \quad[54,2 \%]$ ou quinzenal, $\mathrm{n}=19 \quad[22,9 \%]$ ), sendo a supervisão dos restantes feita com uma periodicidade variável. Em 67,5\% $(\mathrm{n}=56)$ dos casos, o supervisor da Instituição é psicólogo.

\section{Instrumentos}

Inventário de Estilos Supervisivos: Para avaliação dos estilos supervisivos de ambos os supervisores - UM e IE -foi

Psico-USF, v. 12, n. 2, p. 239-248, jul./dez. 2007 utilizada uma versão traduzida do Supervisory Styles Inventory - SSI (Friedlander \& Ward, 1984): o Inventário de Estilos Supervisivos - IES (traduzido por Ribeiro \& Caires, 2006a). Este inventário é composto por 33 itens de auto-relato, distribuídos por 3 subescalas, no qual os orientandos avaliam, numa escala de Likert de 7 pontos, o estilo de supervisão do seu orientador. No âmbito do presente estudo, os dados de duas aplicações do Inventário de Estilos Supervisivos (IES) - uma avaliando o supervisor da UM e outra o supervisor da IE - foram submetidos a análises de componentes principais seguidas de rotação varimax.

Questionário da Qualidade da Supervisão: A versão traduzida do Supervisee Satisfaction Questionnaire - SSQ (Ladany e cols., 1996), o Questionário de Qualidade da Supervisão - QQS (tradução de Ribeiro \& Caires, 2006b), foi utilizada com o intuito de avaliar a satisfação dos orientandos relativamente a vários aspectos da supervisão recebida de ambos os supervisores. Trata-se de um instrumento constituído por 8 itens, avaliados numa escala de Likert de 4 pontos. No presente estudo, foram levadas a cabo duas análises de componentes principais seguidas de rotação varimax, uma para os dados obtidos em relação ao supervisor da UM e outra para o supervisor da IE.

Inventário de Depressão de Beck: Para avaliação da sintomatologia depressiva dos participantes foi utilizado o Inventário de Depressão de Beck - IDB (revisão de 1973, traduzida por T. McIntyre e S. McIntyre, 1995). A resposta a cada um dos itens varia numa escala de Likert de 4 pontos, reflectindo a gravidade da sintomatologia depressiva, podendo o escore total ser de 0 (mínimo) a 63 (máximo). Na versão portuguesa os pontos de corte mais habitualmente usados baseiam-se em dados normativos e sugerem que, de 0 a 9, estamos perante ausência da depressão; de 10 a 15 encaramos uma depressão média, de 16 a 19 observamos uma depressão moderada, de 20 a 29 existe uma depressão moderada a severa e, de 29 em diante, a depressão severa. O estudo de validade realizado por T. McIntyre e Araújo-Soares (1999) com uma amostra de doentes portugueses com dor crónica $(\mathrm{n}=69)$ aponta para um alfa médio de 0,86 . As autoras salientam alguns dos cuidados a ter na interpretação desses valores, atendendo ao tamanho da amostra considerada e à especificidade da população utilizada.

\section{Procedimentos}

Dado que o SSI (Friedlander \& Ward, 1984) e o SSQ (Ladany e cols., 1996) não existiam no contexto português, procedeu-se à sua tradução e retroversão, 
seguidas pelo estudo das suas características psicométricas. Após a tradução dos instrumentos, procedeu-se à sua reflexão falada por um estagiário de cada área de préespecialização, da qual resultaram algumas alterações no nível da linguagem e dos aspectos gráficos.

Os instrumentos foram aplicados pela seguinte ordem: a) Inventário de Estilos Supervisivos (supervisor da UM); b) Questionário da Qualidade da Supervisão (supervisor da UM); c) Inventário de Depressão de Beck; d) Inventário de Estilos Supervisivos (supervisor da IE); e, e) Questionário da Qualidade da Supervisão (supervisor da IE). A introdução do Inventário de Depressão de Beck entre a avaliação de cada supervisor visou evitar efeitos de contaminação, separando de forma clara a avaliação desses dois agentes.

\section{Resultados}

Os dados foram analisados com recurso ao SPSS (versão 14.0). Optou-se pela estratégia aconselhada por Fife-Schaw (2006): levar a cabo análises estatísticas paramétricas e correspondentes não-paramétricas e comparar conclusões. Uma vez que as conclusões retiradas a partir dos dois conjuntos de testes foram as mesmas em todos os casos, serão apresentados os resultados obtidos com os testes paramétricos. Tal opção prende-se a duas razões: são mais robustos e permitem-nos a utilização de análises multivariadas, reduzindo o número de testes $\mathrm{e}$, portanto, da probabilidade do erro tipo I.
Influência do género

Análises preliminares revelaram que o sexo dos estagiários não teve qualquer efeito na forma como estes avaliam os estilos supervisivos do supervisor da UM (Wilks' lambda $=0,97, \mathrm{~F} \quad(3,74)=0,69$, n.s.) nem da IE (Wilks' lambda $=0,96, \mathrm{~F}(3,75)=1,04$, n.s.). Da mesma forma, não foi encontrado efeito do género na satisfação com a supervisão da UM ( $t(77)=-0,18$, n.s.) nem com a da IE $(t(77)=-0,02$, n.s.). Relativamente à sintomatologia depressiva, não foram encontradas diferenças significativas entre estagiários do sexo feminino e os do sexo masculino $(\mathrm{t}(80)=-1,24$, n.s. $)$.

A influência da área de préespecialização: Relativamente à variável "área de pré-especialização", a forma como os orientandos avaliaram os seus supervisores não revelou diferenças significativas no que diz respeito aos estilos supervisivos (UM - Wilks' lambda $=0,88, \mathrm{~F}(9,175.380)=1,03$, n.s.; IE - Wilks' lambda $=0,90, \mathrm{~F}(9,177.813)=0,85$, n.s.), nem à satisfação com a supervisão (UM - $\mathrm{F}(3,75)=0,57$, n.s.; $\mathrm{IE}$ $\mathrm{F}(3,75)=0,48$, n.s.). No entanto, foram encontradas diferenças no nível da sintomatologia depressiva $(\mathrm{F}(3,78)=2,91, \mathrm{p}<0,05)$. O teste post-hoc de Gabriel revelou diferenças marginalmente significativas entre os estagiários da área de pré-especialização em psicologia escolar e da educação (maior sintomatologia depressiva), quando comparados com os estagiários de outras áreas. Perante esses dados, optou-se por tratar a amostra como um todo nas análises subsequentes. Na Tabela 1 apresentam-se as medidas descritivas (média e desvio padrão) dos instrumentos utilizados.

Tabela 1 -Medidas descritivas das subescalas

Média (DP)

Supervisor da UM

1. Estilo supervisivo

Atractivo

$40,96(10,13)$

Orientado para a tarefa

$46,66(11,04)$

Sensível a nível interpessoal

$33,94(8,82)$

2. Satisfação com a supervisão

$19,62(6,16)$
Supervisor da IE
1. Estilo supervisivo
Atractivo
$42,78(11,82)$
Orientado para a tarefa
$47,06(12,19)$
Sensível a nível interpessoal
$34,59(9,43)$
2. Satisfação com a supervisão
$20,89(7,13)$

Sintomatologia depressiva

$7,39(5,57)$ 
Supervisor da Universidade do Minho vs supervisor da instituição de estágio

Comparados os resultados relativos aos supervisores da UM e da IE, não foram encontradas diferenças significativas na forma como ambos foram avaliados em qualquer uma das dimensões (atractivo, $\mathrm{t}(75)=-1,26$, n.s.; orientado para a tarefa, $\mathrm{t}(75)=-0,18$, n.s.; sensível a nível interpessoal, $\mathrm{t}(76)=-0,58$, n.s. e satisfação com a supervisão, $t(75)=-1,26$, n.s.). $\mathrm{Na}$ realidade, foram encontradas correlações entre as apreciações efectuadas pelos orientandos em relação a ambos os supervisores (atractivo, $\mathrm{r}=0,26, \mathrm{p}<0,05$; orientado para a tarefa, $\mathrm{r}=0,51, \mathrm{p}<0,001$; sensível a nível interpessoal, $\mathrm{r}=0,47, \mathrm{p}<0,001$; satisfação com a supervisão, $\quad \mathrm{r}=0,39, \quad \mathrm{p}<0,001)$ e o que revela concordância na forma como ambos os supervisores foram avaliados.

Em relação a cada supervisor, individualmente, observaram-se correlações significativas entre todas as dimensões avaliadas, quer para o supervisor da UM quer para o supervisor da IE. Na Tabela 2 dão-se a conhecer os valores de tais correlações.

Tabela 2 - Intercorrelações entre as diferentes dimensões por supervisor

\begin{tabular}{|c|c|c|c|c|c|}
\hline & & Atractivo & Orient. tarefa & Sensível interp. & Satisfa. superv. \\
\hline \multirow[t]{2}{*}{ Atractivo } & UM & --- & $0,58 * * *$ & $0,67 * * *$ & $0,66^{* * *}$ \\
\hline & Instituição & --- & $0,51 * * *$ & $0,76^{* * *}$ & $0,67 * * *$ \\
\hline \multirow{2}{*}{$\begin{array}{l}\text { Orientado } \\
\text { tarefa }\end{array}$} & UM & & --- & $0,82 * * *$ & $0,78^{* * *}$ \\
\hline & Instituição & & --- & $0,76^{* * *}$ & $0,68^{* * *}$ \\
\hline \multirow{2}{*}{$\begin{array}{l}\text { Sensivel } \\
\text { interpessoal }\end{array}$} & UM & & & --- & $0,76 * * *$ \\
\hline & Instituição & & & --- & $0,72 * * *$ \\
\hline \multirow{2}{*}{$\begin{array}{l}\text { Satisfação } \\
\text { c/ supervisão }\end{array}$} & UM & & & & --- \\
\hline & Instituição & & & & --- \\
\hline
\end{tabular}

$* * * \mathrm{p}<0,001$

As elevadas correlações existentes entre os estilos orientado para a tarefa e sensível a nível interpessoal relativamente a ambos os supervisores permitem-nos inferir que a adopção de uma postura "mista" conjugando a vertente mais técnica, objectiva e instrumental do acompanhamento dos estágios com alguma sensibilidade para com as necessidades e dificuldades do estagiário - emerge como a combinação de estilos que maiores níveis de satisfação gera entre os estagiários.

\section{Influência da periodicidade da supervisão}

Procurando averiguar a influência da periodicidade da supervisão no modo como os estagiários avaliam os estilos supervisivos, no seu grau de satisfação com a supervisão e na sintomatologia depressiva apresentada, recorreu-se a testes de diferenças tomando os 59 estagiários cuja supervisão decorreu com uma periodicidade regular (semanal ou quinzenal) e os 19 para quem tal supervisão foi menos sistemática. Os restantes 5 alunos não deram informação sobre esta variável. As análises preliminares revelaram que a periodicidade (regular vs. irregular) da supervisão tem apenas impacto em relação ao supervisor da UM. A sua influência surge no nível da forma como os estagiários avaliam os estilos supervisivos (Wilks' lambda $=0,81, \mathrm{~F}(3,74)=5,64, \mathrm{p}<0,01$ ), $\mathrm{e}$ na sua satisfação com a supervisão $(\mathrm{t}(77)=-2,78, \mathrm{p}<0,01)$. Relativamente à sintomatologia depressiva, os dados revelam a ausência de qualquer influência da periodicidade/regularidade da supervisão (Tabela 3). 
Tabela 3 - Diferenças entre as avaliações do supervisor da UM e da IE

\begin{tabular}{|c|c|c|c|c|c|c|}
\hline & \multicolumn{3}{|c|}{ Supervisor da UM } & \multicolumn{3}{|c|}{ Supervisor da IE } \\
\hline & $\begin{array}{c}\text { Regular } \\
\text { Média (DP) } \\
(\mathrm{N}=59)\end{array}$ & $\begin{array}{c}\text { Irregular } \\
\text { Média (DP) } \\
(\mathrm{N}=19)\end{array}$ & $\mathrm{F}(1,76)$ & $\begin{array}{l}\text { Psicólogo } \\
\text { Média (DP) } \\
(\mathrm{N}=54)\end{array}$ & $\begin{array}{l}\text { Não-psic. } \\
\text { Média (DP) } \\
(\mathrm{N}=22)\end{array}$ & $\mathrm{F}(1,74)$ \\
\hline Estilos supervisivos & & & & & & \\
\hline Atractivo & $40,42(11,27)$ & $42,53(5.62)$ & 0,61 & $43,93(10,76)$ & $38,82(13,98)$ & $2,95^{* * *}$ \\
\hline Orientado para a tarefa & $48,63(10,42)$ & $41,16(11.23)$ & $7,11 *$ & $47,80(12,41)$ & $45,91(12,04)$ & 0,37 \\
\hline Sensível a nível interp. & $34,75(8,84)$ & $31,68(8.88)$ & 1,72 & $35,96(8,90)$ & $30,91(10,02)$ & $4,69 * *$ \\
\hline & $\begin{array}{c}\text { Média (DP) } \\
\quad(\mathrm{N}=61)\end{array}$ & $\begin{array}{c}\text { Média (DP) } \\
\quad(\mathrm{N}=18)\end{array}$ & $\mathrm{t}(77)$ & $\begin{array}{c}\text { Média (DP) } \\
(\mathrm{N}=54)\end{array}$ & $\begin{array}{c}\text { Média (DP) } \\
\quad(\mathrm{N}=22)\end{array}$ & $\mathrm{t}(74)$ \\
\hline
\end{tabular}

\begin{tabular}{llllll}
\hline Satisfação com a superv. & $20,62(6,18)$ & $16,22(4,85)$ & $-2,78^{*}$ & $21,70(7,53)$ & $18,68(5,67)$
\end{tabular}

\begin{tabular}{|c|c|c|c|c|c|c|}
\hline & $\begin{array}{c}\text { Média (DP) } \\
\quad(\mathrm{N}=63)\end{array}$ & $\begin{array}{c}\text { Média (DP) } \\
(\mathrm{N}=19)\end{array}$ & $\mathrm{t}(80)$ & $\begin{array}{c}\text { Média (DP) } \\
(\mathrm{N}=55)\end{array}$ & $\begin{array}{c}\text { Média (DP) } \\
\quad(\mathrm{N}=24)\end{array}$ & $\mathrm{t}(77)$ \\
\hline Sintomatologia depres. & $7,65(6,01)$ & $6,53(3,81)$ & $-0,77$ & $8,05(5,98)$ & $6,00(4,44)$ & 1,51 \\
\hline
\end{tabular}

${ }^{*} \mathrm{p}<0,01 ;{ }^{* *} \mathrm{p}<0,05 ;{ }^{* *} \mathrm{p}<0,10$

Os testes de diferenças revelam que os estagiários que têm uma supervisão regular avaliam o seu supervisor da UM como sendo mais orientado para a tarefa do que aqueles cuja supervisão é irregular $(\mathrm{F}(1,76)=7,11, \mathrm{p}<0,01)$. Tais percepções fazem todo o sentido atendendo ao estilo de supervisor a que se reportam: exaustivo, centrado, estruturado. As análises revelam, também, que os orientandos cuja supervisão é irregular estão menos satisfeitos com a supervisão $(t(77)=-2,78, p<0,01)$.

No que se refere a eventuais efeitos diferenciadores da satisfação dos estagiários relativamente à supervisão recebida, à avaliação dos estilos supervisivos e aos sintomas depressivos apresentados em razão da área de formação do supervisor da IE (psicólogo vs. não-psicólogo), os testes de diferenças revelaram um efeito marginalmente significativo da profissão do supervisor na forma como os estagiários avaliam os estilos supervisivos (Wilks' lambda $=0,91, \mathrm{~F}(3,72)=2,37, \mathrm{p}<0,10)$, bem como a sua satisfação com a supervisão $(t(74)=1,69, \mathrm{p}<0,10)$. No que se refere ao estilo supervisivo, os resultados mostram que os orientandos avaliam os seus supervisores como mais sensíveis a nível interpessoal quando estes são psicólogos $(\mathrm{F}(1,74)=4,69, \mathrm{p}<0,05)$. Relativamente à satisfação com a supervisão, esta é maior nos casos em que o supervisor é psicólogo.

\section{Predição da satisfação com a supervisão}

Para predição da satisfação com a supervisão, foram realizadas duas análises de regressão múltipla: uma para predizer a satisfação com a supervisão da UM (Tabela 4a) e outra para predição da satisfação com a supervisão da IE (Tabela 4b).

Para a primeira análise de regressão foram usados como preditores: os estilos supervisivos do orientador, a periodicidade da supervisão e a sintomatologia depressiva do estagiário (Tabela 4a). Dado que uma análise de multicolinearidade revelou problemas, retirou-se a subescala Sensível a Nível Interpessoal. Retirou-se, ainda, um dos participantes pelo facto de se ter verificado que constituía um outlier. 
Tabela 4a - Modelo de regressão para predição da satisfação com supervisor UM

\begin{tabular}{lcccc}
\hline (a) Supervisão na UM & $\mathrm{R}^{2}\left(\mathrm{R}^{2}\right.$ ajustado $)$ & $\mathrm{F}(4,70)$ & $\beta$ & $\mathrm{t}$ \\
\hline Periodicidade da supervisão & & & 0,19 & $2,92^{* * *}$ \\
Atractivo & $0,77(0,76)$ & $58,68^{* * *}$ & 0,42 & $5,50^{* * *}$ \\
Orientado para a tarefa & & & 0,50 & $6,31^{* * *}$ \\
Sintomatologia depressiva & & $-0,02$ & $-0,38$ \\
\hline
\end{tabular}

$* * * \mathrm{p}<0,001$

Os dados relativos à supervisão na UM permitemnos concluir que o modelo encontrado é significativo $(\mathrm{F}(4,70)=58,68, \mathrm{p}<0,001)$, explicando $77 \%$ da variância. $\mathrm{Ou}$ seja, os maiores níveis de satisfação dos estagiários em relação ao supervisor da UM estão associados à regularidade na supervisão $(\beta=0,19, \mathrm{t}=2,92, \mathrm{p}<0,001)$ e a um estilo atractivo $(\beta=0,42, t=5,50, p<0,001)$ e orientado para a tarefa $(\beta=0,50, t=6,31, p<0,001)$.
Relativamente à satisfação com a supervisão na IE, usaram-se como preditores os estilos supervisivos do orientador, a sua profissão e também a sintomatologia depressiva do estagiário (Tabela 4b). Uma vez mais, uma análise de multicolinearidade revelou problemas, pelo que a subescala sensível a nível interpessoal foi retirada.

Tabela 4b - Modelo de regressão para predição da satisfação com supervisor IE

\begin{tabular}{lcrcc}
\hline (b) Supervisão na instituição & $\mathrm{R}^{2}\left(\mathrm{R}^{2}\right.$ ajustado $)$ & $\mathrm{F}(4,69)$ & $\beta$ & $\mathrm{t}$ \\
\hline Profissão do supervisor & & & $-0,09$ & $-1,12$ \\
Atractivo & $0,60(0,58)$ & $25,70^{* * *}$ & 0,43 & $4,52^{* * *}$ \\
Orientado para a tarefa & & & 0,43 & $4,72^{* * *}$ \\
Sintomatologia depressiva & & & $-0,01$ & $-0,13$ \\
\hline
\end{tabular}

${ }^{* * *} \mathrm{p}<0,001$

Tal como é possível verificar, o modelo encontrado é significativo $(F(4,69)=25,70, \quad p<0,001)$, explicando $60 \%$ da variância. Tal significa que uma maior satisfação dos estagiários em relação ao supervisor da IE está associada ao estilo atractivo $(\beta=0,43, \mathrm{t}=4,52, \mathrm{p}<0,001)$ e orientado para a tarefa $(\beta=0,43, t=4,72, p<0,001)$. Uma vez mais a sintomatologia depressiva não aparece como preditor da satisfação dos formandos em relação à supervisão assegurada.

\section{Síntese e comentários finais}

O presente estudo pretendeu conhecer as percepções de um grupo de estagiários de psicologia da Universidade do Minho relativamente ao acompanhamento assegurado pelos seus supervisores da universidade e da instituição de estágio. À semelhança do observado noutros estudos (Glickman \& Bey, 1990; Wallace, 1991), os resultados encontrados apontam a (ir)regularidade da supervisão como uma variável diferenciadora da forma como os estagiários avaliam os estilos supervisivos e a sua satisfação com o supervisor da UM. Assim, nos casos em que a supervisão foi feita num registo regular - correspondente a cerca de $3 / 4$ do grupo estudado - os estagiários percepcionaram o seu supervisor como predominantemente orientado para a tarefa e foram maiores os seus níveis de satisfação relativamente ao acompanhamento assegurado.

Relativamente à área de formação do supervisor da instituição (psicólogo vs. não-psicólogo), os níveis de satisfação foram maiores perante supervisores psicólogos, sendo estes percebidos como mais "sensíveis a nível interpessoal" (e.g. mais perspicazes, intuitivos, "terapêuticos"). Tratando-se essa sensibilidade de uma ferramentachave quanto à intervenção psicológica, e com previsíveis benefícios para a própria relação supervisiva, a sua ausência ou carácter menos "apurado" pareceu não responder tão adequadamente às necessidades dos estagiários. Comparando as apreciações feitas aos supervisores da UM e da IE, observou-se que, para ambos os supervisores, os maiores níveis de satisfação surgiram associados a um perfil que combina o estilo "atractivo" e o "orientado para a tarefa", ao qual acresce, no caso dos supervisores da UM, uma frequência regular.

Os dados aqui sintetizados fornecem pistas de grande relevância à reflexão e intervenção sobre a supervisão dos estágios em geral, e sobre a formação inicial de psicólogos em particular. Em primeiro lugar, sinalizam algumas questões essenciais a considerar aquando da organização dos estágios, tendo em vista a potenciação das oportunidades formativas encerradas por esta etapa e a prevenção de algumas dificuldades que 
possam emergir. Entre estas últimas salienta-se a ausência de um profissional da mesma área do estagiário no contexto de intervenção. A par das questões associadas à inserção na instituição e à sua socialização profissional, a ausência de momentos de observação de um modelo profissional, acompanhada de prática, feedback, reforço e monitorização, poderá circunscrever as possibilidades de crescimento e aprendizagem desses formandos.

$\mathrm{Na}$ impossibilidade de assegurar um profissional de psicologia na instituição de estágio, exige-se um acompanhamento mais próximo e regular por parte do supervisor da universidade, podendo este traduzir-se, por exemplo, numa maior frequência das visitas à instituição, objetivando um conhecimento mais aprofundado $\mathrm{e}$ contextualizado das suas dinâmicas, uma melhor articulação com o supervisor local e, também, um maior apoio a este último.

Entretanto, mesmo assegurando a presença de um psicólogo no local de estágio, mantém-se a relevância de um acompanhamento regular pelo supervisor da universidade, conjugando as necessidades instrumentais e formativas dos estagiários com as do foro mais emocional. A sua complementaridade com o acompanhamento no local de estágio é, claramente, a solução mais desejável. O apoio recebido em cada uma das instituições supre necessidades distintas, não sendo, por isso, qualquer uma delas substituível (Cardoso, 1999; McIntyre, Hagger \& Wilkin, 1994). Para além do mais, é fundamental um entendimento prévio entre supervisor da universidade, da instituição de estágio e o formando relativamente aos objectivos do estágio e critérios de avaliação; aos objectivos, formato e periodicidade da supervisão; e aos papéis e responsabilidades a assumir por cada um dos elementos desta tríade.

A potenciação desse acompanhamento - quer na sua assunção isolada quer em complemento com o outro parceiro formativo -, implica, também, a cuidadosa selecção das instituições e dos profissionais que colaboram com a universidade. A detenção de condições materiais, científicas e humanas ajustadas às necessidades dos estagiários e, também, ao programa de formação da universidade surgem como critérios essenciais na selecção desses parceiros (Ellsworth \& Albers 1995). Relativamente a este último, e na senda das reflexões já largamente difundidas na literatura (mas com pouca concretização prática), defende-se como essencial que a formação profissionalizante dos estudantes do ensino superior envolva parcerias entre a universidade e os contextos laborais, baseados no desenvolvimento de uma cultura de formação interinstitucional partilhada e fundamentadora de um projecto de formação sólido, coerente e ajustado às necessidades do "terreno" e às dos diferentes agentes implicados. Reforçando alguns dos elementos centrais discutidos ao longo do presente trabalho, acrescentaríamos uma abordagem que alie a vertente objectiva, pragmática e formal deste projecto comum a uma vertente mais aberta, "genuína" e "aceitante" do outro, visando, com esta, atingir soluções mais criativas, profícuas e potencialmente promotoras de maiores (e melhores) níveis de crescimento das entidades envolvidas e dos seus agentes (e.g. académicos, profissionais do "terreno"), bem como da qualidade dos seus "produtos" (e.g. técnicos formados nas instituições do ensino superior, suas intervenções e implicações das mesmas no bem-estar e satisfação dos seus clientes).

\section{Referências}

Alarcão, I. \& Tavares, J. (2003). Supervisão da prática pedagogica: uma perspectiva de desenvolimento e aprendizagem $\left(2^{\mathrm{a}}\right.$ ed.). Coimbra: Almedina.

Beck, A. T., Steer, R. A. \& Garbin, M. G. (1988). Psychometric properties of the Beck Depression Inventory: Twenty-five years of evaluation. Clinical Psychology Review, 8, 77-100.

Caires, S. (2001). Vivências e percepcõés do estágio no ensino superior. Braga: Universidade do Minho, Grupo de Missão para a Qualidade do Ensino/Aprendizagem Apontamentos UM.

Caires, S. (2003). Vivências e percepções do estágio pedagógico: a perspectiva dos estagiários da Universidade do Minho. Tese de Doutorado. Braga: Universidade do Minho - Instituto de Educação e Psicologia.

Caires, S. \& Almeida, L. S. (2001). O estágio como um espaço de desenvolvimento de competências pessoais e profissionais: o papel da supervisão. Em A. Gonçalves, L. S. Almeida, R. M. Vasconcelos \& S. Caires (Orgs.). Actas de comunicacões científicas. Seminário da Universidade para o Mundo de Trabalho: Desafios para um Diálogo (pp. 227246). Braga: Universidade do Minho.

Capel, S. A. (1997). Changes in students' anxieties and concerns after their first and second teaching practices. Educational Research, 39(2), 211-228.

Cardoso, F. (1999). A articulação entre as práticas de orientação de estágio dos orientadores de escola $e$ as dos orientadores da universidade. Tese de Mestrado. Aveiro: Universidade de Aveiro - Departamento de Ciências da Educação.

Dicionário de Língua Portuguesa (2003). Porto: Porto Editora.

Ellis, M. V. (2001). Harmful supervision, a cause of alarm: Comment on Gray et al. (2001) and Nelson and Friedlander (2001). Journal of Counseling Psychology, 48(4), 401-406.

Psico-USF, v. 12, n. 2, p. 239-248, jul./ dez. 2007 
Ellsworth, J. \& Albers, C. M. (1995). Tradition and authority in teacher education reform. Em H. Petrie (Org.). Profissionalization, partnership and power: Building professional development schools (pp. 159-176). New York: Sate University of New York Press.

Fife-Schaw, C. (2006). Levels of measurement. Em G. M. Breakwell, S. Hammond, C. Fife-Schaw \& J. A. Smith (Orgs.). Research methods in psychology ( $3^{\text {rd }}$ ed.). London: Sage.

Friedlander, M. F. \& Ward, L. G. (1984). Development and validation of the supervisory styles inventory. Journal of Counseling Psychology, 31(4), 541-557.

Glickman, C. D. \& Bey, T. M. (1990). Supervision. Em W. R. Houston (Org.). Handbook of research on teacher education (pp. 549-566). New York: Macmillan.

Hawkey, K. (1997). Roles, responsibilities, and relationships in mentoring: A literature review and agenda for research. Journal of Teacher Education, 48(5), 325-335.

Ladany, N., Hill, C., Corbett, M. \& Nutt, E. (1996). Nature, extent and importance of what psychotherapy trainees do not disclose to their supervisors. Journal of Counselling Psychology, 43(1), 10-24.

Malglaive, G. (1997). Formação e saberes profissionais: entre a teoria e a prática. Em R. Canário (Org.). Formação e situações de trabalho (pp. 53-60). Porto: Porto Editora, Colecção Ciências da Educação.

Matos, P. M. \& Costa, M. E. (1993). Supervisão em psicoterapia e aconselhamento psicológico: uma experiência em contexto universitário. Cadernos de Consulta Psicológica, 9, 19-28.

McIntyre, H. Hagger \& M. Wilkin (1994). Mentoring: Perspectives of school-based teacher education. London: Kogan Page.

McIntyre. T. \& McIntyre, S. (1995). Inventário de Depressão de Beck - versão portuguesa. Braga: Universidade do Minho.
McIntyre, T. \& Araújo-Soares, V. (1999). Inventário da Depressão de Beck: um estudo de validade numa amostra de doentes com dor crónica. Em APPORT (Org.). Actas de comunicações científicas. VI Conferência Internacional de Avaliação Psicológica: Formas e Contextos (pp. 245-255). Braga: APPORT.

Nelson, M. L. \& Friedlander, M. L. (2001). A close look at conflictual supervisory relationships: The trainee's perspective. Journal of Counseling Psychology, 48(4), 384-395.

Ribeiro, A. C. \& Caires, S. (2006a). Inventário de Estilos Supervisivos - tradução para a língua portuguesa do Supervisory Styles Inventory de Friedlander e Ward (1984). Versão não publicada.

Ribeiro, A. C. \& Caires, S. (2006b). Questionário da Qualidade da Supervisão - tradução para a língua portuguesa do Supervisee Satisfaction Questionnaire; de Ladany, Hill, Corbett e Nutt (1996). Versão não publicada.

Ryan, G., Toohey, S. \& Hughes, C. (1996). The purpose, value and structure of the practicum in the higher education: A literature review. Higher Education, 31, 355377.

Schlossberg, N. K. (1989). Overwhelmed: Coping with life's ups and downs. Lexington, MA: Lexington Books.

Soares, I. M. C. (1995). Supervisão e inovação numa perspectiva construtivista do desenvolvimento. Em I. Alarcão (Org.). Supervisão de professores e inovação educacional (pp. 135-147). Aveiro: CIDInE.

Wallace, M. J. (1991). Training foreign language teachers: A reflective approach. Cambridge: Cambridge.

Recebido em maio de 2007 Reformulado em agosto de 2007 Aprovado em setembro de 2007

Sobre as autoras:

Ana Catarina Ribeiro de Figueiredo é licenciada em Psicologia, na área de pré-especialização em Psicologia Clínica e da Saúde, pela Universidade do Minho. O presente artigo decorre do seu trabalho de pesquisa realizado no âmbito do estágio curricular, orientado pelas restantes co-autoras.

Susana Caires Fernandes é doutora em Psicologia da Educação pela Universidade do Minho, professora auxiliar desta universidade, lecionando disciplinas de Psicologia da Educação nos cursos graduados e pós-graduados de 
Formação de Professores e Psicologia. Tem realizado vários trabalhos de pesquisa sobre os estágios, transição para o mundo de trabalho e supervisão.

Carla C. E. Martins é doutorada em Psicologia do Desenvolvimento pela Universidade de Reading (UK) e especializada no domínio da metodologia quantitativa da investigação psicológica. É professora auxiliar da Universidade do Minho, sendo responsável pelas disciplinas de Seminário de Investigação e Métodos Quantitativos de Análise de Dados nos cursos graduados e pós-graduados de Psicologia.

Vera Lúcia M. Ramalho é mestre em Psicologia Clínica e da Saúde pela Universidade do Minho e diretora de um centro de consulta psicológica e apoio educativo no seio do qual realiza a sua prática clínica e de investigação. 